\title{
An Optimized Scheduling Model for Multi-skilled Air Traffic Controllers Based on Fatigue Analysis
}

\author{
Miao-zhuang Xu, Nian-cheng Zhang, Ao-bo Lv, Bo-han Xu, Chao Li, Tian-xu Liu, Run-qing \\ Zhang, Zhi-yuan Shen ${ }^{+}$
}

College of Civil Aviation, Nanjing University of Aeronautics and Astronautics, Nanjing, China, 210016

\begin{abstract}
In recent years, the number of air insecurity incidents caused by fatigue factors has been increasing. As one of its important components, the shortage of controllers in regional airports has also attracted more attention. As the situation of "one person with multiple positions" in regional airports, it is necessary to optimize the scheduling model of multi-skilled Air Traffic Controller. In this work, a scheduling model of Multi-skilled air traffic controllers scheduling model based on fatigue analysis is proposed. Firstly, the fatigue model and risk level are established. Then 13 Constraint conditions in scheduling problems are listed. A genetic algorithm is used to solve the scheduling model for controllers, meteorologists, technical monitor officers based on the consideration of fatigue. The case study shows that the proposed scheduling model meets the needs of the regional airport. It can also save human resources costs and reduce controller fatigue to achieve the maximum benefit.
\end{abstract}

Keywords: regional airports, air traffic controllers, fatigue, scheduling model

\section{Introduction}

With the rapid development of China's civil aviation industry, the number of flights has increased rapidly, the level of fatigue of staff has continued to increase. According to statistics, in 2018, the number of small and medium-sized airports with less than 5 million domestic passenger throughput in China has reached 188, accounting for $80 \%$ of the total number of airports in China. However, the number of control personnel in small and medium-sized airports does not match it, and one person has multiple positions including meteorology, communications guidance, and intelligence. Civil aviation service departments tend to be diversified, and the demand for multi-skilled scheduling is becoming increasingly prominent. In practice, most managers rely on personal experience and intuition to schedule and assign multi-skilled personnel, which makes it difficult to ensure the rationality of project human resource use [1]. The frequency of changes in the air transport market continues to accelerate, and empirical scheduling methods have been unable to adapt to market changes. Therefore, on the basis of considering the fatigue of controllers, how to optimize the staffing of multiple departments, solve the scheduling problem of mixed-skilled personnel efficiently, and carry out scientific and reasonable scheduling is particularly important.

Research on fatigue of controllers is relatively extensive in foreign countries. NASA reports that there are 52,000 unsafe incidents caused by fatigue, accounting for $21 \%$ of the total. Research on human resource flexibility is also earlier. For example, Albert Corominas pointed out in 2005 that the company's profit will increase with the increase of the company's human resource flexibility. Nor does it affect this conclusion [2].

In China, Zhang Xingjian and Yuan Leping, who found that fatigue and tension will allow controllers to complete the same task requires more control frequency response [3]. Li ming and $\mathrm{Xu} \mathrm{Zhe}$ pointed out that

Corresponding author. Tel.: + 8613951916587; fax: +025-84893461.

E-mail address: shenzy@nuaa.edu.cn. 
scheduling and assign multi-skilled persons involved in project activities and time scheduling multi-skilled personnel assigned to these two issues [4]. At this stage, the controller scheduling method has been optimized to a certain extent. For example, Fang Li based on the existing duty problem of Xiamen Air Traffic Control Station and optimized the work characteristics of each unit to obtain the final duty plan [5].Zhang Jiao carried out a correlation analysis on the two schemes of upper two days off and upper one day off,founding that the former is better [6].

In the study of Zhen LIU's "Quantitative Evaluation Model of Fatigue Risk Based on the Voice Response of Controllers" [7], by studying the voice response of controllers, a model of controller fatigue risk was established by means of probability statistics. Based on the results of this research, this article will expand on related introductions.

\section{Controller Fatigue}

\subsection{Definition and Effects}

Fatigue is a common phenomenon at work and in life. It is a psycho-physiological state in which mental or / and physical activity capacity and effectiveness are reduced due to excessive mental effort, physical effort, or illness. It is usually accompanied by discomfort and a desire to rest, Subjective experience with reduced motivation [8]. Insufficient sleep, sleep-wake rhythm disorders, and excessive mental activity can cause drowsiness, lethargy, or subjective fatigue, which is called mental fatigue, and is characterized by burnout, decreased alertness, lack of motivation, decreased cognitive activity, and decreased work efficiency [9].Among them, in terms of behavioral activities, the reaction ability is liable to decrease under fatigue conditions, the controller cannot effectively monitor the request or recitation of the crew, and cannot give instructions quickly and accurately in emergency situations, or even give wrong instructions, and the probability of accident increase. Therefore, it is of great positive significance to take fatigue factors into consideration when scheduling controllers.

\subsection{Establishment of Fatigue Model}

Through a series of studies and analysis, we have found that the most direct and effective way to solve the fatigue problem of control personnel at work is to quantify the fatigue analysis and perform systematic fatigue risk management and evaluation. Among them, the quantitative identification of the fatigue state of controllers, the assessment of fatigue risks, and the establishment of the controller's Air Traffic Controller-Fatigue Risk Management System (ATC-FRMS) have become one of the development trends and urgent problems to be solved in the civil aviation management industry [10].

\subsubsection{Calculation of Fatigue Risk}

In the controller's fatigue risk management process, it is in turn identification of hazards, risk assessment, and risk mitigation. This model is mainly used for risk assessment based on data analysis. The main factors are the magnitude of the possibility of risk occurrence and the magnitude of the loss after risk occurrence. From that perspective, If $R_{F T}$ is the risk value of the fatigue event; $P_{F T}$ is the possibility of the fatigue event; $C_{F T}$ is the severity of the fatigue event, then $R_{F T}=P_{F T} \times C_{F T}$ for a given normal probability density distribution function $\mathrm{f}(\mathrm{x})$, If the average response time of the control officer during a certain duty period is $\mu_{t}$, the closer this value is to overall expectations, the less likely the controller is to have a risk of fatigue. Among them, the overall expected value of the distribution function $\mu$ is the axis of symmetry, $\mu_{t}$ is the regional probability of distance, $P\left(\mathrm{x} \in\left[2 \mu-\mu_{t}, \mu_{t}\right]\right)$ to indicate the possibility of a fatigue event for the controller. Therefore, the calculation formula of $P_{F T}$ is:

$$
\begin{aligned}
& \left\{\begin{array}{l}
P_{F T}=\left(\frac{2}{\sqrt{2 \pi} \sigma} \int_{-\infty}^{\mu_{t}} \exp \left(-\frac{(x-\mu)^{2}}{2 \sigma^{2}}\right) d x\right)-1, \mu_{t}>\mu \\
P_{F T}=0, \mu_{t} \leq \mu
\end{array}\right. \\
& \text { Among them, } 0<P_{F T}<1 .
\end{aligned}
$$


Expressed by the degree of deviation between the response time mean $\mu_{t}$ of the control personnel and the overall expected value $\mu$ during a certain duty period. The further away from the overall expected value $\mu$, the more severe the fatigue event. The formula for calculating the severity is:

$$
\begin{aligned}
& C_{F T}=\frac{\mu_{t}-\mu}{\sigma}, \mu_{t}>\mu \\
& C_{F T}=0, \mu_{t} \leq \mu \\
& \text { Among them, } C_{F T}>0 .
\end{aligned}
$$

\subsubsection{Fatigue Risk Level}

According to the nature of the normal distribution curve, $99.7 \%$ of the data will fall within 3 standard deviations of the overall expected value, Therefore, most values of $C_{F T}$ are between 0 and 3. At the same time, according to the $3 \sigma$ principle of the normal distribution, that is:

$$
\begin{aligned}
& P(\mu-\sigma<X \leq \mu+\sigma)=68.3 \% \\
& P(\mu-2 \sigma<X \leq \mu+2 \sigma)=95.4 \% \\
& P(\mu-3 \sigma<X \leq \mu+3 \sigma)=99.7 \%
\end{aligned}
$$

Reasonably divide the respective value intervals of $P_{F T}$, and classify the fatigue risk level according to $R_{F T}=P_{F T} \times C_{F T}$, as shown in Table 1:

Table 1: Fatigue risk level

\begin{tabular}{cccccc}
\hline Risk level & I(No fatigue) & II(Mild fatigue) & III(Fatigue) & IV(Severe fatigue) & V(Excessive fatigue) \\
\hline$P_{F T}$ & $0 \sim 0.68$ & $0.68 \sim 0.95$ & $0.95 \sim 0.99$ & $0.99 \sim 0.999$ & $>0.999$ \\
$C_{F T}$ & $0 \sim 1$ & $1 \sim 2$ & $2 \sim 3$ & $>3$ & $>3$ \\
$R_{F T}$ & $0 \sim 0.68$ & $0.68 \sim 1.98$ & $1.98 \sim 2.97$ & $2.97 \sim 3$ & $>3$ \\
\hline
\end{tabular}

According to the value range of $R_{F T}$, the fatigue risk level of control personnel is divided into 5 levels. When the risk level is I $\sim$ III, the controller believes that the fatigue risk of the controller is within the acceptable range during the current working period; when the risk level is IV or V, the control unit should appropriately intervene on the controller on duty, Take measures such as rest or shifts to effectively reduce the fatigue risk of the control officer.

When the controller fatigue problem is considered in conjunction with the controller scheduling optimization problem, the controller fatigue level can be divided according to the length of the controller's continuous duty time and matched with the controller's fatigue risk level, as shown in Table 2.

Table 2: Fatigue risk level about time

\begin{tabular}{cccccc}
\hline $\begin{array}{c}\text { hours within two } \\
\text { consecutive days } \\
(\mathrm{h})\end{array}$ & $0 \sim 8$ & $8 \sim 12$ & $12 \sim 14$ & $14 \sim 20$ & $>20$ \\
\hline$R_{F T}$ & $0 \sim 0.68$ & $0.68 \sim 1.98$ & $1.98 \sim 2.97$ & $2.97 \sim 3$ & $>3$ \\
Risk level & I(No fatigue) & II(Mild fatigue) & III(Fatigue) & IV(Severe fatigue) & $\begin{array}{c}\text { V(Excessive } \\
\text { fatigue) }\end{array}$ \\
\hline
\end{tabular}

\section{Scheduling Model}

\subsection{Constraints in Scheduling Problems}

This article is aimed at the controllers of small and medium airports. The main control method is procedural control, and most of the shifts are shifted by multiple shifts. Therefore, before establishing a controller scheduling model, set the following key assumptions:

I. One day controller $\mathrm{m}$ ( $\mathrm{m}$ takes 3 or 4 ) shifts, except for the last shift, the rest of the shift is 6 hours each. After-flight duty time is (T-6 (m-1), T is the airport opening time);

II. There are 2 shifts of meteorologists, the first shift is the duty time (t), and the second shift is the supervision time (24-t);

III. Intelligence officer m- 1 shifts, each shift is the same as the controller, but there is no duty after the flight; 
In addition to the mandatory restrictions in "CCAR-93-R5" and "Interim Measures for Civil Aviation to Implement Unscheduled Working Hours and Comprehensive Calculation of Working Hours", considering the impact of fatigue is another important factor considered in this article. Human factors are an important factor affecting aviation safety management, which directly affects the safety of air transport. Among them, the effect of controller fatigue is particularly important. According to the research results of Zhen LIU's "Quantitative Evaluation Model of Fatigue Risk Based on Voice Response of Controllers" in 2018, it is believed that when the risk level is in the I to III level, the controller's fatigue risk is within the acceptable range during the current working time.

According to the actual situation of different small and medium airports, the number of people on duty each time is different, and the following constraints are established:

$\mathrm{HC1}$ : The controller's continuous duty of the control seat must not exceed 6 hours;

HC2: The number of controllers in the control seat should not be less than $P_{c}$ in each shift;

HC3: The duty of any controller in a calendar week shall not exceed 40 hours;

HC4: When the risk level is I $\sim \mathrm{III}$, the controller believes that the fatigue risk of the controller is within the acceptable range during the current working period;

HC5: The controlling unit shall arrange a rest period of at least 24 consecutive hours for the controller within any consecutive 7 calendar days;

HC6: Persons who have both a meteorological license and a regulatory license must not work more than 40 hours a week;

HC7: Only those with meteorological license, the total working time of a week should not exceed 40 hours;

HC8: The number of meteorologists in each weather shift must not be less than $P_{m}$;

HC9: People who have both a weather license and a regulatory license can only schedule one shift at a time;

HC10: Persons who have both an information license and a control license must not work more than 40 hours a week;

HC11: Only persons with intelligence licenses, the total working hours of a week must not exceed 40 hours;

HC12: The number of intelligence officers in each intelligence shift must not be less than $P_{f}$;

HC13: Persons who have both an information license and a control license can only schedule one shift at a time.

\subsection{Setting of Parameters}

\begin{tabular}{|c|c|}
\hline Parameter & Meaning \\
\hline$X_{i j k}$ & $\begin{array}{l}\text { Means that the i-th controller arranges the k-th shift on the j-th day, the duty is represented as } 1 \text {, } \\
\text { and the rest is represented as } 0 \text {; (the same below) }\end{array}$ \\
\hline$X_{c^{\prime}} m_{i j k}$ & Means that meteorologist \& controller, on duty at the control post; \\
\hline$X_{c m^{\prime}}{ }_{i j k}$ & Means that meteorologist \& controller, on duty at the meteorological post; \\
\hline$X_{c^{\prime} f_{i j k}}$ & Means that intelligence officer \& controller who is on duty at the control post; \\
\hline$X_{c f^{\prime} i j k}$ & Means that an intelligence officer \& controller who is on duty at intelligence posts; \\
\hline$X_{c_{i j k}}$ & Means that a pure controller, on duty in a controlled position; \\
\hline$X_{m_{i j k}}$ & Mean pure meteorologists, on duty at meteorological posts; \\
\hline$X_{f_{i j k}}$ & Means pure intelligence officer, on duty at intelligence posts; \\
\hline$I=\{1,2, \cdots, \mathrm{n}\}$ & Represents a collection of $n$ controllers; \\
\hline$I_{c}=\left\{1,2, \cdots, \mathrm{n}_{1}\right\}$ & Represents $\mathrm{n}_{1}$ pure controller set; \\
\hline$I_{\mathrm{m}}=\left\{1,2, \cdots, \mathrm{n}_{2}\right\}$ & Represents a collection of $\mathrm{n}_{2}$ pure weathermen; \\
\hline$I_{f}=\left\{1,2, \cdots, \mathrm{n}_{3}\right\}$ & Represents a collection of $n_{3}$ pure intelligence officers; \\
\hline$I_{c f}=\left\{1,2, \cdots, \mathrm{n}_{4}\right\}$ & Represents a collection of $\mathrm{n}_{4}$ intelligence and control personnel; \\
\hline$I_{c m}=\left\{1,2, \cdots, \mathrm{n}_{5}\right\}$ & Represents a collection of $\mathrm{n}_{5}$ meteorological and regulatory personnel; \\
\hline$J=\{1,2, \cdots, q\}$ & Represents a collection of $q$ days in a scheduling cycle; \\
\hline$K=\{1,2, \cdots, \mathrm{m}\}$ & Represents a collection of $\mathrm{m}$ shifts of control positions in a day; \\
\hline
\end{tabular}




\begin{tabular}{cl}
\hline$K_{m}=\{1,2\}$ & Represents the assembly of 2 shifts of meteorological posts in a day; \\
$K_{f}=\{1,2, \cdots, \mathrm{m}-1\}$ & Represents the collection of $\mathrm{m}-1$ shifts of intelligence posts in a day; \\
$T$ & Represents the opening hours of the airport on that day; \\
$t$ & Represents the first shift duty of a meteorological post; \\
$P_{c}$ & Represents the number of controllers required in a certain period of time; \\
$P_{m}$ & Represents the number of meteorologists required within a certain period of time; \\
$P_{f}$ & Represents the number of intelligence officers needed within a certain period of time; \\
\hline
\end{tabular}

\subsection{Model Establishment}

The goal of the personnel scheduling problem in this article is to rationally arrange the shift time within a scheduling cycle, so that the number of personnel required is minimum and the fatigue level of the personnel can be controlled at a relatively safe level under the relevant conditions. In this way, the purpose of ensuring flight safety, improving personnel job satisfaction, and reducing departmental labor costs are achieved.

According to the analysis and setting of the constraints of the scheduling problem, the constraints can be transformed into the following specific constraint formulas under the parameters established:

$$
\begin{aligned}
& \sum_{\mathrm{k}}^{\mathrm{k}+1} X_{i j k} \leq 1, \forall i \in I, \forall j \in J, \forall k \in K \\
& \sum_{\mathrm{i}=1}^{\mathrm{n}} X_{i j k} \geq P_{c}, \forall i \in I, \forall j \in J, \forall k \in K \\
& \sum_{\mathrm{j}}^{\mathrm{j}+6} \sum_{\mathrm{k}=1}^{\mathrm{m}-1} 6 X_{i j k}+0.6[T-6 \times(\mathrm{m}-1)] \sum_{\mathrm{j}}^{\mathrm{j}+6} X_{i j m} \leq 40, \forall i \in I, \forall j \in\{1,2 \ldots q-6\} \\
& \sum_{\mathrm{j}}^{\mathrm{j}+1} \sum_{\mathrm{k}=1}^{\mathrm{m}-1} 6 X_{i j k}+0.6[T-6 \times(\mathrm{m}-1)] \sum_{\mathrm{j}}^{\mathrm{j}+1} X_{i j m} \leq 14, \forall i \in I, \forall j \in\{1,2 \ldots q-1\} \\
& \prod_{\mathrm{j}=1}^{\mathrm{j}+6}\left(\sum_{\mathrm{k}}^{\mathrm{m}} X_{i j k}\right)=0, \quad \forall i \in I, \forall j \in\{1,2, \ldots q-6\} \\
& \sum_{\mathrm{j}}^{\mathrm{j}+6} \sum_{\mathrm{k}=1}^{\mathrm{m}-1} 6 X_{c^{\prime}} m_{i j k}+\sum_{\mathrm{j}}^{\mathrm{j}+6} 0.6[T-6(m-1)] X_{c^{\prime}} m_{i j m}+\sum_{\mathrm{j}}^{\mathrm{j}+6}\left[t X_{c m^{\prime}}+0.6(24-t) X_{c m^{\prime}}+{ }_{i j 2}\right] \leq 40 \\
& \forall i \in I_{c m}, \forall j \in\{1,2 \ldots q-6\} \\
& \sum_{\mathrm{j}}^{\mathrm{j}+6}\left[t X_{m_{i j 1}}+0.6(24-t) X_{m_{i j 2}}\right] \leq 40, \forall i \in I_{m}, \forall j \in\{1,2 \ldots, q-6\} \\
& \sum_{i \in I_{m}} X_{m_{i j k}}+\sum_{i \in I_{c m}} X_{c m^{\prime}}{ }_{i j k} \geq P_{m}, \quad \forall j \in J, \forall k \in K_{m} \\
& \min \left\{X_{c m^{\prime} i j 1}, \sum_{\mathrm{k}=1}^{2} X_{c^{\prime}} m_{i j k}\right\}=0, \quad \forall i \in I_{c m}, \forall j \in J \\
& (\mathrm{~m}-4)\left(X_{c m^{\prime} i j 2} \times \sum_{\mathrm{k}=\mathrm{m}-1}^{\mathrm{m}} X_{c^{\prime}} m_{i j k}\right)+(\mathrm{m}-3)\left(X_{c m^{\prime}{ }^{\prime} 2} \times \sum_{\mathrm{k}=\mathrm{m}-2}^{\mathrm{m}} X_{c^{\prime}} m_{i j k}\right)=0, \forall i \in I c m, \forall j \in J, m \in\{3,4\} \\
& \sum_{\mathrm{j}}^{\mathrm{j}+6} \sum_{\mathrm{k}=1}^{\mathrm{m}-1} 6 X_{c^{\prime}} f_{i j k}+\sum_{\mathrm{j}}^{\mathrm{j}+6} 0.6[T-6(m-1)] X_{c^{\prime}}^{\prime} f_{j i j m}+\sum_{\mathrm{j}}^{\mathrm{j}+6} \sum_{\mathrm{k}=1}^{\mathrm{m}-1} 6 X_{c f^{\prime}} \leq 40, \quad \forall i \in I_{c f}, \forall j \in\{1,2 \ldots, q-6\} \\
& \sum_{\mathrm{j}}^{\mathrm{j}+6} \sum_{\mathrm{k}=1}^{\mathrm{m}-1} 6 X_{f_{i j k}} \leq 40, \forall i \in I_{f}, \forall j \in\{1,2 \ldots, q-6\} \\
& \sum_{i \in I_{f}} X_{f_{i j k}}+\sum_{i \in I_{c f}} X_{c f^{\prime}}{ }^{\prime j k} \geq P_{f}, \quad \forall j \in J, \forall k \in K_{f} \\
& \min \left\{X_{c f^{\prime} i j k}, X_{c^{\prime}} f_{i j k}\right\}=0, \forall i \in I_{c f}, \forall j \in J, \forall k \in K_{f}
\end{aligned}
$$

them, (4) to (8) are restrictions on controllers, (9) to (12) are restrictions on meteorologists and meteorologists and controllers, and (13) to (16) are restrictions on intelligence officers and intelligence officers and controllers. 


\section{Case Study}

\subsection{Data Analysis}

Aiming at the model proposed in this paper, YULIN Airport will be used as a case for verification analysis. According to the data obtained from the survey, there are 4 pure control personnel, 0 pure intelligence personnel, 9 pure meteorologists, 6 intelligence\&control personnel, and 0 meteorological \& control personnel (ie, $\mathrm{n}_{1}=4, \mathrm{n}_{2}=9, \mathrm{n}_{3}=0, \mathrm{n}_{4}=6, \mathrm{n}_{5}=0$ ). The airport is open for 19 hours, the first shift of the weather crew is 13 hours, the scheduling cycle is 30 days, and the control post has 3 shifts a day (ie, $T=19, t=13, q=30, \mathrm{~m}$ $=3$ ). The minimum number of persons required to control, meteorological, and intelligence posts in the same shift is not less than 2,2, and 1 (ie, $P_{c}=2, P_{m}=2, P_{f}=1$ ). To simplify the calculation process, we can set the shift cycle to 7 days, and then repeat the restoration $(\mathrm{q}=7)$.

\subsection{Results and Analysis}

On the above algorithm and verification based on model parameters, the results obtained are as follows:

The abscissa of the Fig. 1(a) is the difference between the time when a person is scheduled and the longest schedule specified in the regulations during a week. If the value is positive, it means that the staff shift time exceeded the regulatory limit during the week; if the value is negative, it means that the staff shift time did not reach the maximum length specified in the regulations during the week. The black part is the result of manual scheduling. It can be seen that in the case of manual scheduling, a large number of personnel overtime occur, and there is a large unevenness in the scheduling time of personnel, which is a problem for the overall control team. The fairness of regulations and regulatory security pose a great threat. The blue part is the result of using genetic algorithm for scheduling. It can be seen that when using genetic algorithm for scheduling, there is no overtime of personnel scheduling, and the scheduling time of personnel is relatively balanced, which is beneficial to reducing the Overall fatigue.

Further, in order to determine whether the model algorithm can meet the actual running requirements, the complexity, performance ratio, and stability of the algorithm are analyzed, refer to Fig. 1(b).

The abscissa of the graph is the number of iterations, and the ordinate is the relative quality of the calculation result. It can be seen that with the increase of the number of iterations, the quality of the optimal value solution shows a rapid improvement first, and then gradually stabilizes, while the quality of the general solution also shows a rapid rise and then stabilizes. And it is found that the number of iterations of the algorithm to obtain a better solution is within the acceptable range, so the algorithm meets the requirements of practical use.

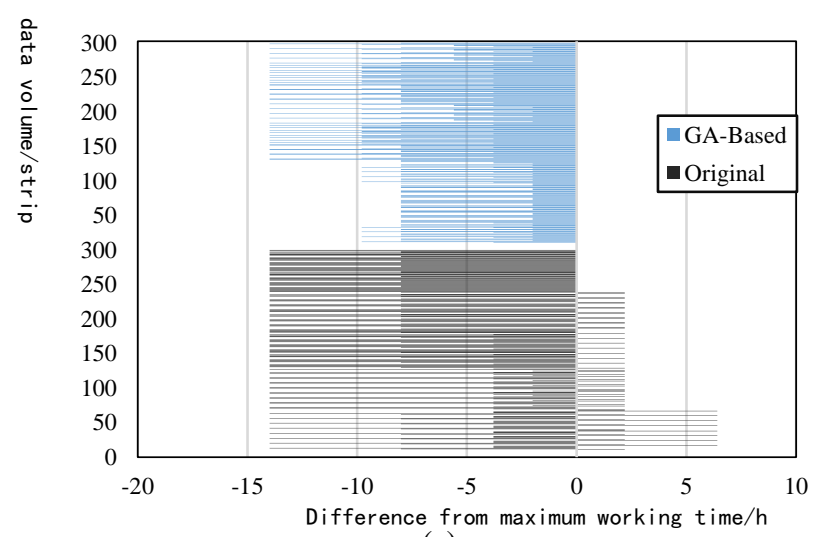

(a)

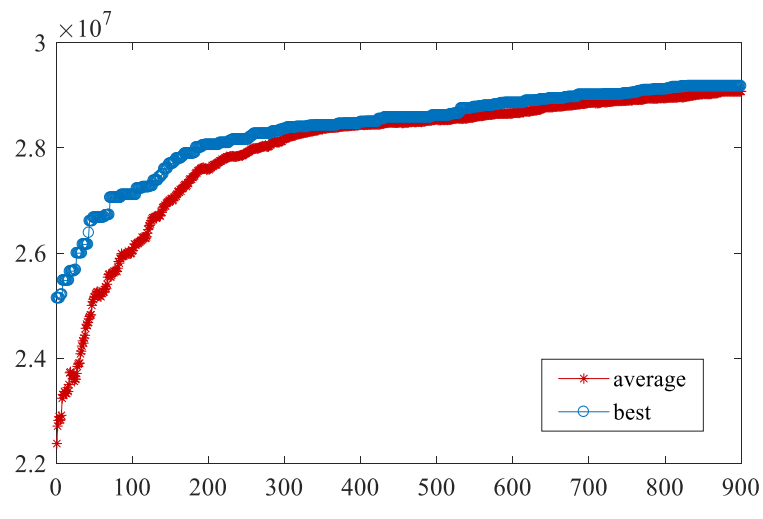

(b)

Fig. 1: The simulation results

\section{Conclusions}

According to the algorithm of this model, the number of violations and fatigue risks of control personnel can be reduced to $0 \%$. It analyzes the multi-skilled scheduling problem of controllers in small and medium airports, and can adjust parameters according to the actual conditions of different small and medium airports 
Great applicability. However, for intelligence personnel and meteorological personnel, the methods for avoiding fatigue have not yet been summarized, which has certain shortcomings. However, considering its applicability, it still has great reference significance for solving the problem of shortage of personnel in small and medium-sized airports in China.

\section{Acknowledgement}

The authors would like to thank the financial support by the Open Fund of 2019 Innovation Training Program of Nanjing University of Aeronautics and Astronautics (2019CX00711).Meantime, thanks to the support from another project of the Fund (2019CX07005).

\section{References}

[1] ACU AST,JURISTON,MORENO AM. Emphasizing human capabilities in software development [J] .Software,2006,23(2):94-101.

[2] Albert Corominas, Amaia Lusa,Rafael Pastor. Using a MILP model to establish a framework for an annualised hours agreement[J]. European Journal of Operational Research,2005,177(3).

[3] Zhang Xingjian, Yuan Leping, Zhao Yifei. Research on speech response characteristics of fatigue and tension controllers [J]. Chinese Journal of safety Sciences, 2018,28 (06): 37-42

[4] Li Ming, Xu Zhe. Project multi skill human resource assignment and scheduling method based on Balanced Optimization [J]. Industrial engineering, 2016,19 (01): 108-114

[5] Fang Li. Study on Optimization of air traffic controller duty scheme in Xiamen air traffic control station [D]. Xiamen University, 2018

[6] Zhang Jiao, sun Ruishan, Li Jingqiang. A comparative study on the shift system of controllers in different regions of China [J]. Occupation and health, 2015,31 (24): 3510-3513

[7] Liu Zhen. Quantitative evaluation model of fatigue risk based on controller voice response time [J]. Science and technology and innovation, 2018 (08): 37-39

[8] Zhang Ying, Chen Chen, Liu Zhenhua, Hu Siyuan. Fatigue state monitoring of psychomotor vigilance task under different perceptual loads [J]. Psychological innovation, 2018,38 (04): 377-384

[9] Ruan Yi, Gu Wei, Ling Changquan. Research progress of mental fatigue assessment method [J]. Journal of the Second Military Medical University, 2019,40 (01): 79-85

[10] Yang Changqi, Tan Juan, Zhou Jingli. Research on fatigue risk management system of air traffic controllers [J]. China Science and technology information, 2017 (17): 61-63 + 16 